\title{
O-GIcNAC and aging: C. elegans as a genetic model to test O-GICNAC roles in type II diabetic insulin resistance
}

\author{
Keith Vosseller \\ Drexel University College of Medicine, Department of Biochemistry and Molecular Biology, Philadelphia, PA \\ 19102, USA
}

Commentary on: Rahman M, Stuchlick O, El-Karim EG, Stuart R, Kipreos ET, and Wells L. Intracellular protein glycosylation modulates insulin mediated lifespan in C. elegans. Aging 2010; 2: this issue.

E-mail: keith.vosseller@drexelmed.edu

A growing body of literature implicates the intracellular post-translational carbohydrate modification O-GlcNAc in type II diabetic insulin resistance $[2,4,12,13]$. OGlcNAc levels are responsive to increased nutrient flux through the hexosamine biosynthetic pathway. Hyperglycemia in type II diabetes thus drives abnormal elevation of O-GlcNAc [1]. Mice over-expressing OGT (the enzyme which catalyzes addition of O-GlcNAc to proteins) display insulin resistant type II diabetic phenotypes [9]. However, OGT appears to be fundamentally essential for mammalian cellular life [10], limiting the potential to test models of O-GlcNAc involvement in insulin resistance/signaling by OGT knock-out studies in mammals.

C. elegans has a conserved insulin-like signaling pathway which regulates phenotypes including life span, dauer larvae formation, stress responses, and macronutrient storage. Additionally, there is a high level of conservation between $C$. elegans and mammalian OGT and OGA (enzyme that removes OGlcNAc) $[3,8]$. In contrast to mammalian systems, $C$. elegans with OGT and OGA null mutant alleles are viable and fertile $[5,3]$. Thus, $C$. elegans is potentially amenable to genetic testing of interactions between O-GlcNAc cycling and insulin signaling/phenotypes. In C. elegans, attenuation of insulin-like signaling induces a so-called dauer larval stage. An insulin receptor-like mutant allele (daf-2) with reduced signaling induces dauer formation, and this was enhanced by an OGA null mutant which elevated O-GlcNAc [3], and inhibited by an OGT null mutant which reduced O-GlcNAc levels [5]. Those results suggested that O-GlcNAc negatively regulates insulin-like signaling in the context of dauer formation. This is reminiscent of the model in which increased hexosamine flux and elevated O-GlcNAc are linked to type II diabetic insulin resistance (reduced insulin signaling) in mammals. Similar regulation of entry into the dauer state induced by a synthetic pheromone called daumone was observed in C. elegans with OGT and OGA inactivating mutant alleles [6]. Additionally, C. elegans OGA mutants display macronutrient storage phenotypes that parallel those of mammalian diabetic states [3].

In this issue, Rahman et al. [11] extend genetic studies of OGT and OGA inactivation in C. elegans to provide important information about specificity of O-GlcNAc regulation of insulin-like phenotypes and the steps in insulin-like signaling that are regulated by O-GlcNAc. Attenuation of insulin-like signaling extends lifespan in C. elegans. Rahman et al. find that OGA inactivating mutants increase life span, while OGT inactivating mutants decrease life span. Similar observations were previously reported [7] and will be discussed in more detail below. The genetic studies of O-GlcNAc cycling linked to longevity indicate elevation of O-GlcNAc inhibits, while decreased O-GlcNAc increases insulinlike signaling, again consistent with models of elevated O-GlcNAc linked to mammalian insulin resistance, and highlighting the potential significance of $C$. elegans as a genetic model to continue examining how O-GlcNAc cycling impinges on insulin signaling. Indeed, Rahman et al. go on to show the long lived OGA null mutant did not further extend long lived phenotypes of conserved insulin signaling homologue mutants of the insulin receptor (daf-2), and downstream signaling components PI 3-kinase (AGE-1), and AKT (SGK-1), consistent with O-GlcNAc regulation acting in this insulin-like pathway. The daf-2 longevity phenotype is dependent on daf-16, a foxO homologue transcription factor that is activated upon attenuation of insulin-like 
signaling. Rahman et al. find that the OGA lifespan extension phenotype is also dependent on daf- 16 . However, they observe that both OGA and OGT null mutants increased daf-16 nuclear localization. This result combined with the observation that OGA and OGT null mutants have opposite affects on longevity, suggest that O-GlcNAc regulation of insulin-like signaling connected to longevity acts downstream of daf-16.

Rahman et al. also examined OGT and OGA null mutant interaction with other known insulin-like signaling dependent phenotypes and found that the OGA mutant was resistant to oxidative stress, while the OGT mutant reduced stress resistance in a daf- 2 mutant background. This is reminiscent of the previously reported affects of OGT and OGA null mutants on UV stress tolerance [7]. Rahman et al. go on to show that the OGA null mutant does not alter known daf-2 (insulin receptor mutant) phenotypes of increased thermal tolerance, delayed developmental timing defects, or reduced fecundity. Since these phenotypes are dependent on daf-16, the results further suggest that O-GlcNAc regulation is occurring downstream of daf16. Thus, the results begin to specifically assign OGlcNAc regulation to only a subset of insulin-like signaling phenotypes. Specificity of O-GlcNAc regulation of stress tolerance and not thermal tolerance is supported by their observation that OGA mutants selectively alter transcription profiles associated with oxidative stress [11].

The previous reports on O-GlcNAc regulation of insulin-like signaling in C. elegans and the extension of that knowledge by Rahman et al. in this issue establish an important model system for study of O-GlcNAc regulation of insulin signaling which will help unravel O-GlcNAc links to mammalian insulin resistance. It should be noted that there are a few minor disparities between the Rahman et al. studies [11] and a previous report [7]. Both reports show that OGT null mutants decrease longevity in both wild type and daf-2 long living backgrounds, indicating that O-GlcNAc negatively regulates insulin-like signaling in the context of longevity. Both reports find that OGA null mutants can lead to a life extension phenotype, but under somewhat different circumstances. Rahman et al. find that the OGA null mutant extends lifespan in a wild type background, but not in daf-2, age-1, or sgk-1 long living mutant backgrounds. Love et al. report no affect of OGA null mutant on life span in a wild type background, but an extension of life span in the daf-2 mutant. As mentioned, daf-16 is required for the daf- 2 longevity phenotype. Rahman et al. find that the increased longevity of the OGA null mutants are dependent on daf-16, whereas Love et al. find that OGA null mutant extension of lifespan in the daf-2 background is not dependent on daf-16.Both reports show that OGT null mutants have aberrant constitutive nuclear accumulation of daf-16, but Rahman et al. find that the OGA null mutant also has this phenotype. Distinctions between OGA null mutant affects being dependent on daf-16 and altering daf-16 nuclear localization may be important to reconcile, as those results help define where in the insulin-like signaling pathway that O-GlcNAc regulation occurs. Differences in background strain, back crossing, and temperatures used may possibly contribute to these disparities and RNAi experiments as well as additional ogt and oga alleles should be able to resolve these issues.

Nevertheless, the stage is set now to go further in defining specific mechanisms of how O-GlcNAc regulates insulin-like phenotypes and how modification of specific proteins influence their function in insulinlike signaling. Love et al. previously began to approach these questions by asking where O-GlcNAc marks chromatin and found a bias at promoters that regulate carbohydrate metabolism and chromatin remodeling [7]. Rahman et al. begin to approach this question using proteomics to identify changes in expression of putative O-GlcNAc modified proteins in response to the OGA null mutant, and find interestingly that a high percentage of proteins identified are regulated by insulin signaling and/or contribute to insulin-like signaling phenotypes. These observations provide a foundation for future functional studies in which the lifespan of a worm may help us to understand type II diabetic insulin resistance.

\section{REFERENCES}

1. Akimoto $Y$ et al. Elevation of the post-translational modification of proteins by O-linked $\mathrm{N}$-acetylglucosamine leads to deterioration of the glucose-stimulated insulin secretion in the pancreas of diabetic Goto-Kakizaki rats. Glycobiology. 2007; 17:127-140.

2. Copeland $R$ et al. Cross-talk between GlcNAcylation and phosphorylation: roles in insulin resistance and glucose toxicity. Am J Physiol Endocrinol Metab. 2008; 295:E17-28.

3. Forsythe ME et al. Caenorhabditis elegans ortholog of a diabetes susceptibility locus: oga-1 (O-GlcNAcase) knockout impacts O-GlcNAc cycling, metabolism, and dauer. Proc Natl Acad Sci U S A. 2006; 103:11952-11957.

4. Hanover JA. Glycan-dependent signaling: O-linked Nacetylglucosamine. FASEB J. 2001; 15:1865-1876.

5. Hanover JA et al. A Caenorhabditis elegans model of insulin resistance: altered macronutrient storage and dauer formation in an OGT-1 knockout. Proc Natl Acad Sci U S A. 2005; 102:11266-11271. 
6. Lee $\mathrm{J}$ et al. Regulation of Dauer formation by OGlcNAcylation in Caenorhabditis elegans. J Biol Chem. 285:2930-2939.

7. Love DC et al. Dynamic O-GlcNAc cycling at promoters of Caenorhabditis elegans genes regulating longevity, stress, and immunity. Proc Natl Acad Sci U S A. 107:7413-7418.

8. Lubas WA et al. O-Linked GlcNAc transferase is a conserved nucleocytoplasmic protein containing tetratricopeptide repeats. J Biol Chem. 1997; 272:9316-9324.

9. McClain DA et al. Altered glycan-dependent signaling induces insulin resistance and hyperleptinemia. Proc Natl Acad Sci U S A. 2002; 99:10695-10699.

10. O'Donnell $\mathrm{N}$ et al. Ogt-dependent X-chromosome-linked protein glycosylation is a requisite modification in somatic cell function and embryo viability. Mol Cell Biol. 2004; 24: 1680-1690.

11. Rahman $\mathrm{MM}$ et al. Intracellular protein glycosylation modulates insulin mediated lifespan in C.elegans. Aging. 2010; 2 : this issue

12. Wells L and Hart GW. O-GIcNAc turns twenty: functional implications for post-translational modification of nuclear and cytosolic proteins with a sugar. FEBS Lett. 2003; 546:154158.

13. Wells L et al. Glycosylation of nucleocytoplasmic proteins: signal transduction and O-GlcNAc. Science. 2001; 291:23762378. 